\title{
Calcium Pectinate Beads Formation: Shape and Size Analysis
}

\author{
Boon-Beng Lee ${ }^{1 *}$, Eng-Seng Chan $^{2} \&$ Pogaku Ravindra ${ }^{3}$ \\ ${ }^{1}$ School of Bioprocess Engineering, Universiti Malaysia Perlis, \\ Kompleks Pusat Pengajian Jejawi 3, 02600 Arau, Perlis, Malaysia \\ ${ }^{2}$ Chemical Engineering Discipline, School of Engineering, Monash University Malaysia, \\ Jalan Lagoon Selatan, 46150 Bandar Sunway, Selangor, Malaysia \\ ${ }^{3}$ School of Engineering and Information Technology, Universiti Malaysia Sabah, \\ 88999 Kota Kinabalu, Sabah, Malaysia \\ *Email: bblee@ unimap.edu.my
}

\begin{abstract}
The aim of this study was to investigate the inter-relationship between process variables and the size and shape of pectin solution droplets upon detachment from a dripping tip as well as Ca-pectinate beads formed after gelation via image analysis. The sphericity factor (SF) of the droplets was generally smaller than 0.05 . There was no specific trend between the SF of the droplets and the pectin concentration or the dripping tip radius. The SF the beads formed from high-concentration pectin solutions and a small dripping tip was smaller than 0.05 . The results show that the Reynolds number and Ohnesorge number of the droplets fall within the operating region for forming spherical beads in the shape diagram, with the exception to the lower boundary. The lower boundary of the operating region has to be revised to $O h=2.3$. This is because the critical viscosity for Ca-pectinate bead formation is higher than that of $\mathrm{Ca}$ alginate beads. On the other hand, the radius of the droplets and beads increased as the dripping tip radius increased. The bead radius can easily be predicted by Tate's law equation.
\end{abstract}

Keywords: bead size; bead shape; Ca-pectinate bead; extrusion-dripping method; pectin.

\section{Introduction}

Bioencapsulation is defined as the process of confining bioactive compounds (e.g. microbial cells, enzymes, animal cells, plant cells, antibiotics, etc.) within a matrix in particulate form (i.e. bead or capsule) in order to achieve one or more desirable effects $[1,2]$. The particles are used to immobilize, protect (or stabilize), controlled-release, and/or alter the properties of the bioactive compounds [1].

Alginate is the most widely used biomaterial for particles formation. It can easily form beads with di- or multivalent cations [3]. The gelling process can be carried out under mild conditions, especially when calcium cations are used to

Received April $6^{\text {th }}, 2013$, Revised September $5^{\text {th }}, 2013$, Accepted for publication January $18^{\text {th }}, 2014$.

Copyright (C 2014 Published by ITB Journal Publisher, ISSN: 2337-5779, DOI: 10.5614/j.eng.technol.sci.2014.46.1.5 
induce gelation. Ca-alginate beads have received much attention in research and industrial applications. However, Ca-alginate beads are not chemically stable. They are sensitive to chelators (e.g. phosphate, citrate, lactate) and monocations (e.g. sodium and magnesium) [4], which may be present in the working medium of most bioprocesses. Consequently, the Ca-alginate beads may be destabilized and may eventually dissolve after several repeatable usages or certain periods of usage.

Pectin is a cell wall structural carbohydrate present in all land plants [4,5]. Currently, the commercially available pectin is extracted mainly from citrus peel and apple pomace $[4,5]$. Pectin forms beads through ionotropic gelation with calcium cations, which are similar to alginate [4-6]. Therefore, the application of pectin for bioencapsulation purposes seems to be attractive. In addition, previous studies have shown that Ca-pectinate beads are less sensitive to ions and chemical agents that destabilize Ca-alginate beads [2,4]. In order to enhance the stability of the beads in bioprocess applications, their mechanical strength should be improved, which in turn is influenced by their size and shape [7]. Therefore, it is important to have a bead formation system that is capable of forming mono-dispersed and spherical beads.

Extrusion dripping is the most common technique used to form mono-dispersed and spherical beads, in which the polymeric solution is flowed through a capillary at a low volumetric flow rate and allowed to drip under gravitational force [1]. Recently, a master shape diagram has been developed to reveal the relationship between the process variables and the shape of the Ca-alginate beads formed by the extrusion dripping method [1]. Moreover, a mathematical model has been introduced to predict the Ca-alginate beads [1]. These models could be beneficial to determine the operating variables for Ca-pectinate bead formation by extrusion dripping. The bead shape and size are affected by various process variables (e.g. type of pectin used, concentration of pectin, concentration of cation, etc.) [7-9].

The aim of this study was to investigate the inter-relationship between process variables and the shape and size of the Ca-pectinate beads formed by extrusion dripping. Shape diagram and mathematical size prediction models have been developed for the Ca-alginate bead formation system. The suitability of the diagram and the models for the Ca-pectinate bead formation system was investigated. 


\section{$2 \quad$ Materials and Methods}

\subsection{Amidated Low Methoxy Pectin}

Amidated low methoxy pectin $($ Pectin LM35, degree of esterification $(\mathrm{DE})=$ $27-33 \%, 20-25 \%$ amidated groups) was kindly donated by TIC Gum (USA). All pectin solutions were prepared by dissolving pectin powder in deionised distilled water according to the desired concentrations (\%w/v) with the use of a mixer (IKA, Germany). In this study, the pectin concentrations ranged from 5.0 $\% \mathrm{w} / \mathrm{v}$ to $8.0 \% \mathrm{w} / \mathrm{v}$. The prepared pectin solutions were left for several hours to remove the entrapped air bubbles before they were used for the further experimentation.

\subsection{Measurement of The Physical Properties of Bead Forming Materials}

The density of the pectin solutions was measured using a digital specific gravity meter (Kyoto Electronics Manufacturing Co Ltd, Japan). The surface tension of the pectin solutions was determined by the LCP coefficient method as described in Lee, et al. [10]. The viscosity of the solutions was determined using a viscometer according to the standard procedure (Brookfield Engineering Laboratories, Inc., Model: LV-DV E203, USA).

\subsection{Experimental Set-up of Ca-Pectinate Bead Formation System}

Figure 1 shows the experimental set-up for the formation of pectin solution droplets and Ca-pectinate beads. All studies were conducted at a controlled temperature of $25^{\circ} \mathrm{C}$. Hypodermic needles (Precision Needle, Singapore) with an outer radius ranging from $0.33 \mathrm{~mm}$ to $0.83 \mathrm{~mm}$ were used, after being blunted and shortened to $3 \mathrm{~mm}$. The gelation bath consisted of $1.5 \% \mathrm{w} / \mathrm{v}$ calcium chloride (Mallinckrodt Baker, Mexico) and $0.1 \%$ w/v Tween 80 (Fluka, USA). The collecting distance of the beads was kept at $10 \mathrm{~cm}$. The gelation time of the Ca-pectinate beads was fixed at 30 minutes. An 8-megapixel digital camera (Canon EOS 350D, Japan, with 3 frames per second, up to 14 frame burst performance) was set up $10 \mathrm{~cm}$ below the dripping tip. The digital camera was used to capture images of the pectin liquid drops when falling to the gelation bath via the influence of gravitational force. 


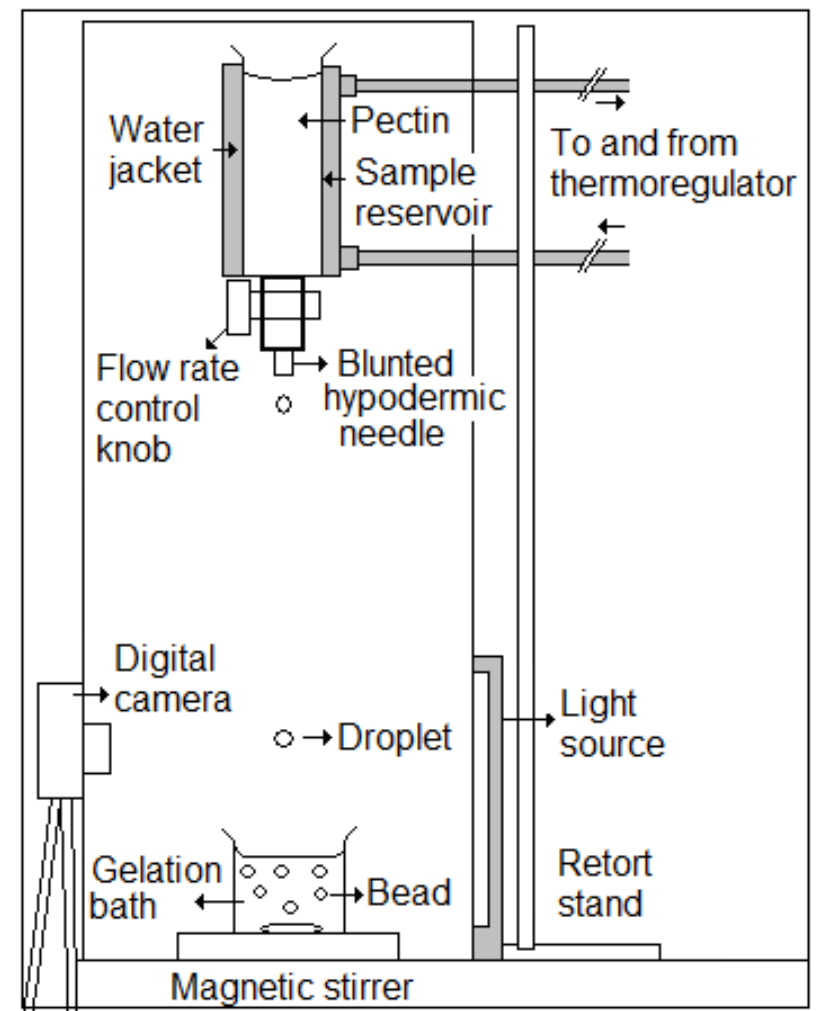

Figure 1 Experimental set-up.

\section{Characterization of Pectin Solution Droplets and Beads}

After gelation, images of the Ca-pectinate beads were captured by the digital camera. The diameter of the solution droplets and beads was determined by an image analyser (SigmaScan Pro 5, SPSS Inc, USA), while the radius of the particles was computed using Microsoft Excel. Since most of the solution droplets underwent elongation during travelling and the beads underwent 'tailing' during gelation, the sphericity factor (SF) was selected as the criterion to quantify the sphericity of the particles [1]. Basically, the SF is defined as:

$$
\mathrm{SF}=\left(\mathrm{d}_{\max }-\mathrm{d}_{\min }\right) /\left(\mathrm{d}_{\max }+\mathrm{d}_{\min }\right)
$$

where, $d_{\max }=$ maximum diameter (pixel), $d_{\min }=$ minimum diameter perpendicular to $\mathrm{d}_{\max }$ (pixel) 
The SF varies from zero for a perfect sphere to approaching unity for an elongated object. Particles with an SF less than 0.05 are considered spherical [1].

\subsection{Statistical Analysis}

The radius and SF of the beads were determined based on the measurement of 30 beads. All standard error bars in the graph were calculated based on a 95\% confidence interval using t-statistic. On the other hand, 10 to 20 samples were taken for droplet analysis.

\subsection{Dimensionless Number Analysis}

The influence of the physical properties of the pectin solution on the sphericity of the Ca-pectinate beads is described by the Ohnersorge number $(\mathrm{Oh})$ [1]. Oh measures the relative importance of viscous force to the surface force of a droplet during falling or impaction in determining the droplet shape:

$$
O h=v \rho /\left(2 \rho r_{d} \gamma\right)^{1 / 2}
$$

where, in the case of this study, $\rho$ is the density of the pectin solution $\left(\mathrm{kg} / \mathrm{m}^{3}\right), \gamma$ is the surface tension of the pectin solution $(\mathrm{mN} / \mathrm{m}), v$ is the kinematic viscosity of the pectin solution $\left(\mathrm{mm}^{2} / \mathrm{s}\right)$, and $r_{d}$ is the radius of the pectin solution droplets $(\mathrm{mm})$.

The influence of droplet impact against the gelation bath on the sphericity of the Ca-pectinate beads is described by the Reynolds number $(R e)$ [1]. Re describes the importance of inertia force to the viscous force of falling solution droplets and is described by the following equation:

$$
R e=2 u r_{d} / v
$$

where, in the case of this study, $u$ is the velocity of the pectin solution droplet $(\mathrm{mm} / \mathrm{s})$ at the point of impact and $v$ is the kinematic viscosity of the pectin solution $\left(\mathrm{mm}^{2} / \mathrm{s}\right)$.

\subsection{Determination of the Velocity of the Droplet at Impact}

In this study, the velocity of the pectin solution droplet at impact $(u)$ was not measured. The falling pectin solution droplet velocity was estimated by a model proposed by Pumphrey and Elmore [11] for rain fall study, as shown in Eq. (4):

$$
u=V_{T}\left[1-\exp \left(\frac{-2 g h}{V_{T}^{2}}\right)\right]^{1 / 2}
$$


where $u$ is the velocity of the pectin solution droplet $(\mathrm{m} / \mathrm{s})$ at the point of impact, $V_{T}$ is the terminal velocity $(\mathrm{m} / \mathrm{s}), g$ is the gravitational acceleration $\left(\mathrm{m} / \mathrm{s}^{2}\right), h$ is the collecting distance $(\mathrm{m})$ (i.e. the distance between dripping tip and gelation bath).

The terminal velocity for spherical droplets was estimated from Atlas's model [12], as shown in Eq. (5):

$$
V_{T}=9.65-10.3 \exp \left(-1.2 r_{d}\right)
$$

\section{$4 \quad$ Results and Discussion}

\subsection{Physical Properties of Pectin Solutions}

Table 1 shows the physical properties of the pectin solutions at various concentrations. As the concentration of pectin increased, the density of the pectin solutions increased slightly, whereas the apparent viscosity at zero shear rate of the solutions increased exponentially. The results are in good agreement with previous studies [13-16]. The surface tension of the pectin solutions decreased from $72.2 \mathrm{mN} / \mathrm{m}$ to $70.8 \mathrm{mN} / \mathrm{m}$ for the solutions with a concentration of $5 \% \mathrm{w} / \mathrm{v}$ to $8 \% \mathrm{w} / \mathrm{v}$, respectively. The results are in good agreement with the theorem of surface thermodynamics (Gibbs adsorption isotherm). This can be explained by the fact that pectin is a hydrophilic polysaccharide hydrocolloid and it has low surface tension activity [16].

Table 1 Physical properties of pectin solutions.

\begin{tabular}{cccc}
\hline $\begin{array}{c}\text { Pectin concentration } \\
(\boldsymbol{\%} \mathbf{w} / \mathbf{v})\end{array}$ & $\begin{array}{c}\text { Density }^{\text {a }} \\
\left(\mathbf{k g} / \mathbf{m}^{\mathbf{3}}\right)\end{array}$ & $\begin{array}{c}\text { Viscosity }^{\text {a }} \\
(\mathbf{m} \text { Pa.s) }\end{array}$ & $\begin{array}{c}\text { Surface tension }^{\text {a }} \\
(\mathbf{m N} / \mathbf{m})\end{array}$ \\
\hline 5 & $1017( \pm 1)$ & $340( \pm 2)$ & 72.2 \\
6 & $1020( \pm 1)$ & $590( \pm 2)$ & 71.6 \\
7 & $1023( \pm 2)$ & $1200( \pm 35)$ & 71.5 \\
8 & $1028( \pm 1)$ & $2110( \pm 34)$ & 70.8 \\
\hline
\end{tabular}

a Measurements were done in replicate and the coefficient of variance (CV) was less than 5\%.

The standard deviation of the samples is shown in parentheses.

\subsection{Shape Analysis of Pectin Solution Droplets and Ca-Pectinate Beads}

Figure 2 shows the SF of the pectin solution droplets and Ca-pectinate beads formed from different pectin concentrations and dripping tip radii. As shown in Figure 2(a), the SF of the pectin solution droplets varied between 0.005 and 0.063 . The droplets were generally spherical before impaction on the surface of the gelation bath. Figure 2(a) clearly illustrates that there was no trend between the SF of the droplets and the pectin concentration or the dripping tip radius. 
However, there was some variation in the droplet SF. We speculate that this variation can be attributed to the variation in the force balance between the surface tension-viscosity of the droplet and the pressure of the air pushing up against the bottom of the droplet as the droplet falls from the dripping tip [1,17].
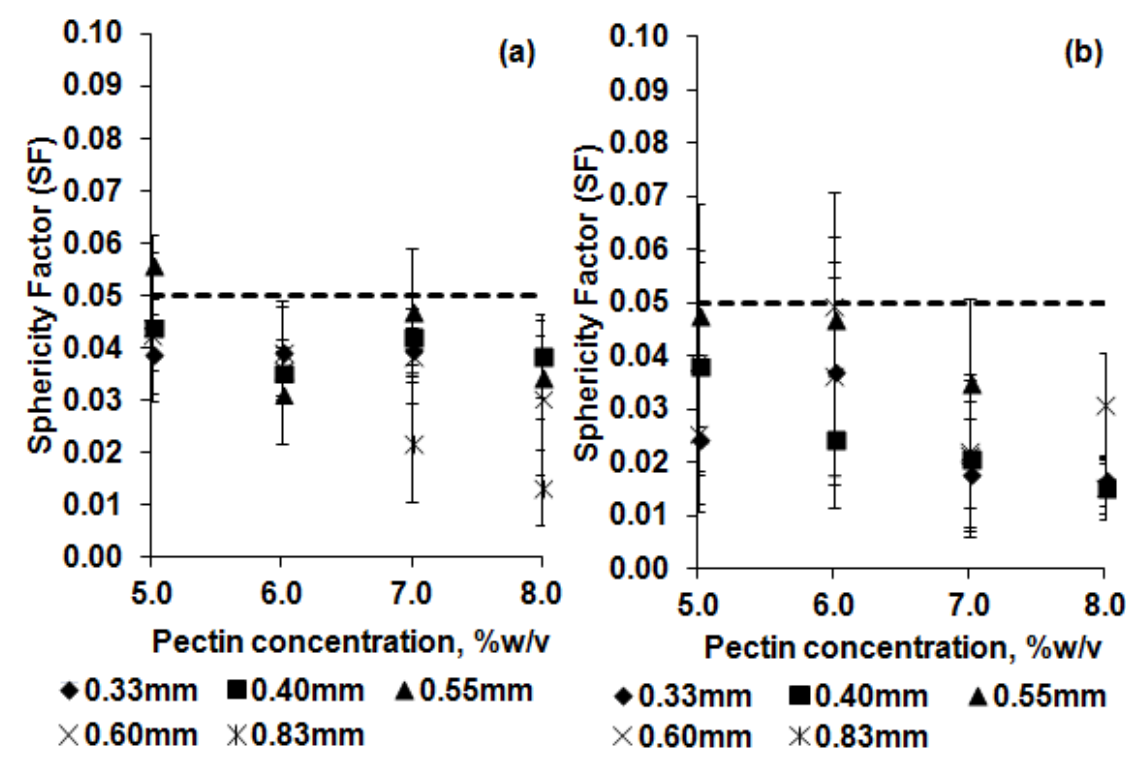

Figure 2 Sphericity factor (SF) of the pectin solution droplets (a) and $\mathrm{Ca}$ pectinate beads (b) formed from different pectin concentrations and dripping tip radii.

Although all the droplets formed before gelation were generally spherical (SF < 0.05), only the beads formed from high pectin concentration solutions (i.e. 7 $\% \mathrm{w} / \mathrm{v}$ and $8 \% \mathrm{w} / \mathrm{v}$ ) were spherical after gelation (see Figure 2(b)). Minor deformation happened to the $5 \% \mathrm{w} / \mathrm{v}$ and $6 \% \mathrm{w} / \mathrm{v}$ pectin solution droplets when impacted on the gelation bath surface. As a result, some of the beads were not spherical after gelation.

Table 2 shows the results of a two-sample t-test to compare the effects of low and high pectin concentrations on the SF of the beads. The results indicate that high pectin concentration solutions ( 7 and $8 \% \mathrm{w} / \mathrm{v})$, which are more viscous (> $1200 \mathrm{~m} \mathrm{Pa.s}$ ), form more spherical beads than solutions of low concentrations (5 and $6 \% \mathrm{w} / \mathrm{v}$ ) (see also Figure 2(b)). This is because the mean SF of the beads produced by high pectin concentration solutions is significantly lower than that of low pectin concentration solutions (P-value $<0.05)$. The results are in good agreement with the results of previous studies on Ca-alginate bead systems $[1,18,19]$. The viscosity of the pectin solution can determine the resistance to 
shape change in the solution droplet during falling as well as when hitting (impaction) and entering the gelling bath [1]. The results of this study suggest that the critical viscosity to form spherical Ca-pectinate beads is about 1200 mPa.s.

Table 2 Statistical analysis of pectin concentration and dripping tip radius effect on bead SF.

\begin{tabular}{ccccc}
\hline Process variables & $\begin{array}{c}\text { No. } \\
\text { Samples }\end{array}$ & Mean & $\begin{array}{c}\text { Standard } \\
\text { deviation }\end{array}$ & P-value $^{\text {a }}$ \\
\hline Pectin concentration: & & & & \\
Low $(5$ and 6 \% w/v) & 480 & 0.0391 & 0.0248 & 0.000 \\
High $(7$ and $8 \% \mathrm{w} / \mathrm{v})$ & 520 & 0.0204 & 0.0121 & \\
Dripping tip radius: & & & & \\
Small $(0.33$ and $0.40 \mathrm{~mm})$ & 400 & 0.0243 & 0.0159 & 0.000 \\
Large $(0.55,0.60$ and $0.83 \mathrm{~mm})$ & 600 & 0.0327 & 0.0238 & \\
${ }^{\mathrm{a}}$ Based on a two-sample t-test (Minitab 15, Minitab Inc., USA) & & &
\end{tabular}

Table 2 also shows the comparison of the effects of small and large dripping tips on the SF of the beads using the two-sample t-test. The results show that the mean SF of the beads produced by small dripping tips was significantly lower than that produced by large dripping tips (P-value < 0.05$)$. In other words, small dripping tips significantly produce more spherical beads as compared to large dripping tips. This is because the droplet detached from a small dripping tip is smaller and lighter, and hence it experiences less impact force when hitting the gelation bath surface [1].

In order to correlate the inter-relationship of surface tension force, viscous force and inertia force of the polymeric solution droplets on the sphericity of polymeric gel beads, a master shape diagram has been developed based on the dimensionless number (i.e. $O h$ and $R e$ ) $[1,20]$. Under the experimental conditions (i.e. pectin concentrations, dripping tip radii, collecting distance) in this study, the $O h$ and $R e$ of the pectin solution droplets was in the range of 0.64.6 and 2.2-16.9, respectively (see Table 3). As the viscosity of the pectin solutions increased, the $O h$ of the droplets increased, whereas the $R e$ of the droplets decreased. The results clearly indicate that the influence of viscous force becomes more dominant than that of surface tension force and inertia force as the viscosity of the pectin solutions increases.

Figure 3 shows the influence of $O h$ and $R e$ of the pectin solution droplets on the sphericity of the Ca-pectinate beads formed under the experimental conditions in this study. The results in this study fall within the boundaries that the authors [1] have proposed to be the operating region for forming spherical Ca-alginate beads. However, the lower boundary of the shape diagram has been revised 
because the Ca-pectinate beads formed from pectin solutions with a concentration less than $7 \% \mathrm{w} / \mathrm{v}$ were not uniformly spherical. Therefore, the lower boundary of the shape diagram for the Ca-pectinate beads formation system is suggested to be $\mathrm{Oh}=2.3$ (see Figure 3).

Table 3 Ohnesorge numbers and Reynolds numbers of pectin solution droplets.

\begin{tabular}{cccc}
\hline $\begin{array}{c}\text { Pectin solution viscosity } \\
(\mathbf{m . P a})\end{array}$ & $\begin{array}{c}\text { Droplet radius } \\
(\mathbf{m m})\end{array}$ & $\boldsymbol{O h}$ & $\boldsymbol{R} \boldsymbol{b}$ \\
\hline $340( \pm 2)$ & $1.49-1.98( \pm 0.01)$ & $0.6-0.7$ & $12.8-16.9$ \\
$590( \pm 2)$ & $1.56-2.01( \pm 0.01)$ & $1.1-1.3$ & $7.1-9.4$ \\
$1200( \pm 35)$ & $1.54-2.04( \pm 0.01)$ & $2.3-2.7$ & $3.5-4.7$ \\
$2110( \pm 34)$ & $1.55-1.98( \pm 0.01)$ & $4.1-4.6$ & $2.2-2.7$ \\
\hline
\end{tabular}

a The standard deviation of the samples is shown in parentheses.

b The standard error, based on a $95 \%$ confidence interval using t-statistic, is shown in parentheses.

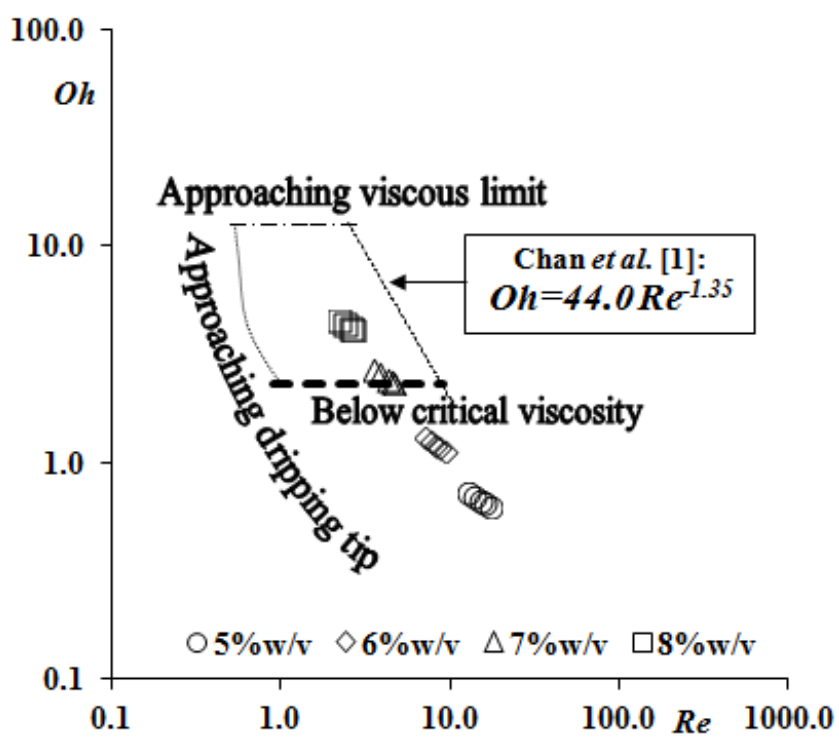

Figure 3 Shape diagram of Ca-pectinate beads formed by the extrusion dripping method. The area within the dotted lines is the proposed operating region for forming spherical beads $(\mathrm{SF}<0.05)$.

\subsection{Size Analysis of Pectin Solution Droplets and Ca-Pectinate Beads}

In this study, the radius of pectin solution droplets and gel beads upon gelation was investigated. Figure 4 shows the radius of pectin solution droplets and $\mathrm{Ca}$ pectinate beads formed from different pectin concentrations and different 
dripping tip sizes. The radius of the droplets and beads increased as the dripping tip radius increased. This is because the droplet grows at the dripping tip nozzle until it reaches its critical dimension, or when gravitational force is no longer balanced by surface tension force, the droplet will detach from the nozzle [21]. The critical dimension of the droplet prior to detachment is approximately proportional to the dripping tip nozzle; this correlation is commonly known as Tate's law [21]. A similar observation was reported in a previous study [22]. In reference to Figure 4, the radius of the pectin solution droplets and the Capectinate beads showed no specific trend of change when the pectin solution concentration increased for a particular dripping tip. This could be due to the influence of surface tension force on the droplet formation of all tested pectin solutions were comparable, as their values are nearly the same (about 71.5 $\mathrm{mN} / \mathrm{m})$.

When the droplet radius and bead radius were compared, no apparent reduction was observed in the particle radius, as illustrated in Figure 4. In fact, the experimental results show that the bead radius slightly increased (about $0.7 \%$ ). The results were in agreement with the previous finding that Ca-pectinate beads were swollen during gelation in $2 \% \mathrm{w} / \mathrm{v}$ calcium chloride solution [23].
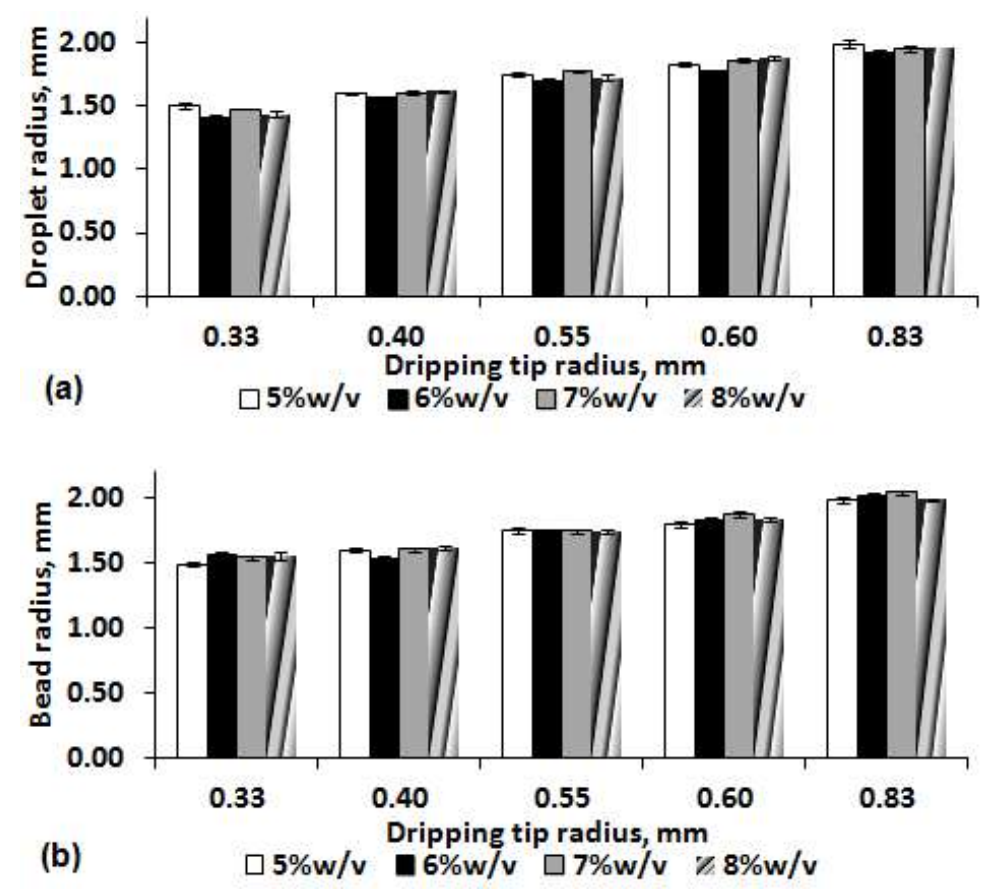

Figure 4 The radius of pectin solution droplets (a) and Ca-pectinate beads (b) formed from different pectin concentrations and dripping tip sizes. 
Commonly, the radius of polymeric gel beads is approximately predicted by the Tate's law equation [20,24]:

$$
r_{b}=(3 r \gamma / 2 \rho g)^{1 / 3}
$$

where, $\rho$ is density $(\mathrm{g} / \mathrm{ml}), \gamma$ is surface tension $(\mathrm{mN} / \mathrm{m}), r$ is the dripping tip radius $(\mathrm{cm}), r_{b}$ is the beads radius $(\mathrm{cm})$, and $g$ is the gravitational force.

Recently, a mathematical model has been introduced by the authors [1] to predict the size of Ca-alginate beads with taking consideration of the residual liquid that remains at the dripping tip after droplet detachment and bead shrinkage. The bead radius prediction model (also called the modified Tate's law equation) is described by Eq. (7).

$$
r_{b}=k_{S} k_{L F}(3 r \gamma / 2 \rho g)^{1 / 3}
$$

where $k_{S}=$ size change factor, $k_{L F}=$ liquid loss factor $\left(k_{L F}=0.98-0.08 r\right)$.

The results from this study were different from the observations made of Caalginate bead formation as reported previously by several researchers $[1,23]$. In this study, the $k_{s}$ is a swelling factor (i.e. 1.007) instead of a shrinkage factor. This could be due to the gel networking interaction between calcium cations with polyguluronate chains in pectin being different from that of alginate [25].

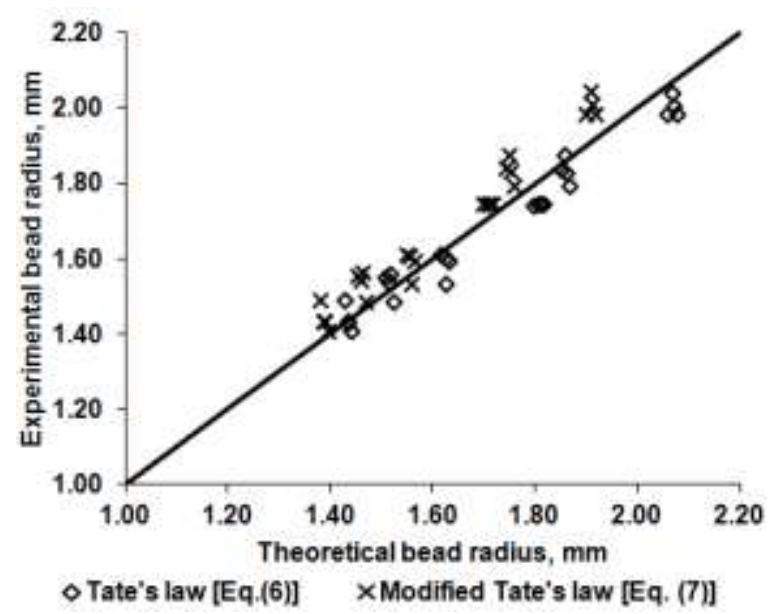

Figure 5 Plot of experimentally determined bead radii against those predicted by the Tate's law equation (Eq. (6)) and the modified Tate's law equation (Eq.(7)).

As shown in Figure 5, the bead sizes predicted by both the Tate's law equation and the modified Tate's law equation were in good agreement with the 
experimental results. An error analysis was conducted on the bead size prediction models; the results are summarized in Table 4. Based on the results, the Tate's law equation in its raw form gave a good prediction, with an average absolute deviation (AAD) of $2.4 \%$ and a maximum absolute deviation (MAD) of 5.6\%. A good prediction was obtained through the liquid loss and bead swelling factors were not considered in the Tate's law equation. This is because the required factors are set off as the Ca-pectinate beads swell after gelation. As a result, the Ca-pectinate bead radius can be easily predicted using the Tate's law equation.

Table 4 Error analysis of bead radius prediction models

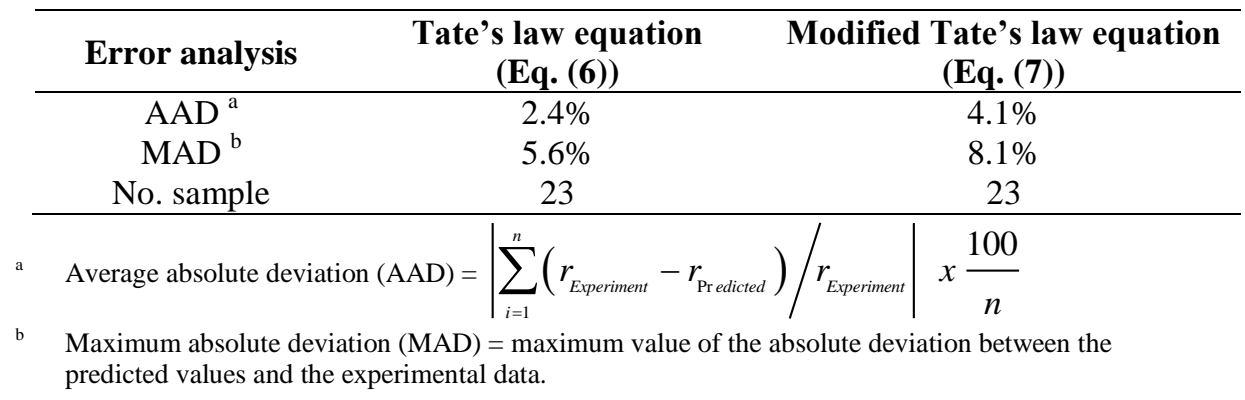

\section{Conclusion}

The pectin solution droplets were generally spherical $(\mathrm{SF}<0.05)$ after detachment from the dripping tip prior to gelation. As a result, spherical $\mathrm{Ca}$ pectinate beads were produced (SF $<0.05$ ). The SF of the droplets had no correlation with the pectin concentration or dripping tip radius. However, the SF of the beads was significantly influenced by the pectin concentration and dripping tip radius. The SF of the beads formed from viscous pectin solutions (i.e. 7 and $8 \% \mathrm{w} / \mathrm{v}$ ) was significantly lower than that of low viscous pectin solutions (i.e. 5 and $6 \% \mathrm{w} / \mathrm{v}$ ). The SF of the beads produced by small dripping tips was significantly lower than that of the beads produced by large dripping tips. The inter-relationships between all process variables and the particle shape were studied using the Ohnesorge number $(O h)$ against the Reynolds number $(R e)$ plot. The $O h$ and $R e$ of the pectin solution droplets were found to fall within the operating region for forming spherical particles of the shape diagram, except the lower boundary was revised to $O h=2.3$. This is because the critical viscosity to form spherical Ca-pectinate beads was found to be about 1200 $\mathrm{mPa}$.s. On the other hand, the bead size analysis showed that the radius of the pectin solution droplets and the beads increased as the dripping tip radius increased. The droplet and bead radius was not dependent on the pectin solution concentration. The droplet and bead radius was well predicted by both the Tate's law equation and the modified Tate's law equation, with an AAD smaller 
than 5\%. The bead radius could be predicted easily by the Tate's law equation without requiring a liquid loss and swelling factor. This is because the $\mathrm{Ca}$ pectinate beads did not shrink after gelation but slightly swelled to the ideal size predicted by the Tate's law equation.

\section{Acknowledgements}

The authors thank Universiti Malaysia Perlis for providing the financial support for this study.

\section{References}

[1] Chan, E.S., Lee, B.B., Ravindra, P. \& Denis, P., Prediction Models for Shape and Size of Ca-alginate Macrobeads Produced through ExtrusionDripping Method, Journal of Colloid and Interface Science, 338(1), pp. 63-72, 2009.

[2] Voo, W.P., Ravindra P., Tey, B.T. \& Chan, E.S., Comparison of Alginate and Pectin based Beads for Production of Poultry Probiotic Cells, Journal of Bioscience and Bioengineering, 111(3), pp. 294-299, 2011.

[3] Dulieu, C., Poncelet, D. \& Neufeld, R.J., Encapsulation and Immobilization Techniques, Cell Encapsulation Technology and Therapeutics, Kuthreiber, W.M., Lanza, R.P. \& Chick, W.L. (eds.) Birkhauser Publisher, Boston, pp. 3-17, 1999.

[4] Strand, B.L., Morch, Y.A. \& Skjak-Braek, G., Alginate as Immobilization Matrix for Cells, Minerva Biotech, 12, pp. 223-33, 2000.

[5] Sundar Raj, A.A., Rubila, S., Jayabalan, R. \& Ranganathan, T.V., A Review on Pectin: Chemistry due to General Properties of Pectin and its Pharmaceutical Uses, Scientific Reports, 1, 550-1-4, 2012.

[6] Gemeiner, P., Nahalka, J., Vikartovska, A., Nahalkova, J., Tamaska, M., Sturdik, E., Markovic, O., Malovikova, A., Zatkova, I. \& Ilavsky, M., Calcium Pectate Gel Could be a Better Alternative to Calcium Alginate Gel in Multiple Applications of Immobilized Cell, Immobilized cells: Basics and Applications, Wijffels, R.H., Buitelaar, R.M., Bucke, C. \& Tramper, J. (eds.), Elsevier Science B.V., pp. 76-83, 1996.

[7] Wong, T.W., Colombo, G. \& Sonvico, F., Pectin Matrix as Oral Drug Delivery Vehicle for Colon Cancer Treatment, AAPS Pharm. Sci. Tech., 12 (1), pp. 201-214, 2011.

[8] Rosinaski, S., Marison, I., Hunkeler, D., Grigorescu, G., Lewinska, D., Ritzen, L.G., Viernstein, H., Teunou, E., Poncelet, D., Zhang, Z., Fan, X. \& Serp, D., Characterization of Microcapsules: Recommended Methods based on Round-Robin Testing, Journal of Mircoencapsulation, 19(5), pp. 641-659, 2002. 
[9] Tho, I., Sande, S.A. \& Kleinebudde, P., Cross-Linking of Amidated Low Methoxylated Pectin with Calcium During Extrusion/Spheronisation: Effect on Particle Size and Shape, Chemical Engineering Science, 60, pp. 3899-3907, 2005.

[10] Lee, B.B., Ravindra, P. \& Chan, E.S., New Drop Weight Analysis for Surface Tension Determination of Liquids, Colloids and Surfaces A: Physicochemical and Engineering Aspects, 332(2-3), pp. 112-120, 2009.

[11] Pumphrey, H.C. \& Elmore, P.A., The Entrainment of Bubbles by Drop Impacts, Journal of Fluid Mechanics, 220, pp. 539-567, 1990.

[12] Saylor, J.R. \& Grizzard, N.K., The Optimal Drop Shape for Vortices Generated by Drop Impacts: The Effect of Surfactants on The Drop Surface, Experiments in Fluids, 36, pp. 783-790, 2004.

[13] Sriamornsak, P., Chemistry of Pectin and Its Pharmaceutical Uses: A Review, Silpakorn University International Journal, 3(1-2), pp. 206-228, 2003.

[14] Zhou, J.H., Wu, Y.X. \& Shen, Z.Q., Viscous-flow Properties and Viscosity-Average Molecular Mass of Orange Peel Pectin, J. Cent. South Univ. Technol., 15(s1), pp. 520-524, 2008.

[15] Brejholt, S.M., Chapter 13: Pectin, Food Stabilisers, Thickeners and Gelling Agents, Imerson, A. (ed.), Blackwell Publishing Ltd. United Kingdom, pp. 237-265, 2009.

[16] Lee, B.-B., Chan, E-.S., Ravindra, P. \& Khan, T.A., Surface Tension of Viscous Biopolymer Solutions Measured using the Du Nouy Ring Method and the Drop Weight Methods, Polymer Bulletin, 69, pp. 471-489, 2012.

[17] Al-Hajry, H.A., Al-Maskry, S.A., Al-Kharousi, L.A., El-Mardi, O., Shayya, W.H. \& Goosen, M.F.A., Electrostatic Encapsulation and Growth of Plant Cell Cultures In Alginate, Biotechnology Progress, 15, pp. 768-774, 1999.

[18] Seifert, D.B. \& Philips, J.A., Production of Small, Monodispersed Alginate Beads for Cell Immobilization, Biotechnology Progress, 13, pp. 562-568, 1997.

[19] Lee, K.Y. \& Heo, T.R., Survival of Bifidobacterium Longum Immobilized in Calcium Alginate Beads in Simulated Gastric Juices and Bile Salt Solution, Applied and Environmental Microbiology, 66(2), pp. 869-873, 2000.

[20] Heinzen, C., Berger, A. \& Marison, I., Use of Vibration Technology for Jet Break-Up for Encapsulation of Cells And Liquids in Monodisperse Microcapsules, Fundamentals of Cell Immobilization Biotechnology, Nedovic V. \& Willaert R. (eds.), Kluwer Academic Publishers, London, pp. 262-263, 2004.

[21] Lee, B.B., Ravindra, P. \& Chan, E.S., A Critical Review: Surface and Interfacial Tension Measurement by Using the Drop Weight Method, Chemical Engineering Communications, 195, pp. 889-924, 2008. 
[22] Navarro, A.R., Rubio, M.C. \& Callier, D.A.S., Production of Ethanol by Yeasts Immobilized in Pectin, Eur. J. Appl. Microbiol Biotechnol., 17, pp. 148-151, 1983.

[23] Bourgeois, S., Gernet, M., Pradeau, D., Andremont, A. \& Fattal, E., Evaluation of Critical Formulation Parameters Influencing The Bioactivity of B-Lactamases Entrapped in Pectin Beads, International Journal of Pharmaceutics, 324, pp. 2-9, 2006.

[24] Poncelet, D., Babak, V.G., Neufeld R.J., Goosen, M.F.A. \& Burgarski, B., Theory of Electrostatic Dispersion of Polymer Solutions in the Production of Microgel Beads Containing Biocatalyst, Advances in Colloid and Interface Science, 79, pp. 213-228, 1999.

[25] Braccini, I. \& Pérez, S., Molecular Basis of Ca2+-Induced Gelation in Alginates and Pectins: the Egg-Box Model Revisited, Biomacromolecules, 2, pp. 1089-1096, 2001. 